\title{
The Role of Gut-Derived Lipopolysaccharides and the Intestinal Barrier in Fatty Liver Diseases
}

\author{
Lingxuan An ${ }^{1}$. Ulrich Wirth ${ }^{1}$. Dominik Koch ${ }^{1} \cdot$ Malte Schirren ${ }^{1} \cdot$ Moritz Drefs $^{1}$ - Dionysios Koliogiannis ${ }^{1}$. \\ Hanno Nie $\beta^{1}$. Joachim Andrassy ${ }^{1}$ - Markus Guba ${ }^{1}$ - Alexandr V. Bazhin ${ }^{1} \cdot$ Jens Werner ${ }^{1}$. Florian Kühn ${ }^{1}$ (i)
}

Received: 4 July 2021 / Accepted: 14 October 2021 / Published online: 3 November 2021

(C) The Author(s) 2021

\begin{abstract}
Background Hepatosteatosis is the earliest stage in the pathogenesis of nonalcoholic fatty (NAFLD) and alcoholic liver disease (ALD). As NAFLD is affecting $10-24 \%$ of the general population and approximately $70 \%$ of obese patients, it carries a large economic burden and is becoming a major reason for liver transplantation worldwide. ALD is a major cause of morbidity and mortality causing $50 \%$ of liver cirrhosis and $10 \%$ of liver cancer related death. Increasing evidence has accumulated that gut-derived factors play a crucial role in the development and progression of chronic liver diseases.

Methods A selective literature search was conducted in Medline and PubMed, using the terms "nonalcoholic fatty liver disease," "alcoholic liver disease," "lipopolysaccharide," "gut barrier," and "microbiome."

Results Gut dysbiosis and gut barrier dysfunction both contribute to chronic liver disease by abnormal regulation of the gut-liver axis. Thereby, gut-derived lipopolysaccharides (LPS) are a key factor in inducing the inflammatory response of liver tissue. The review further underlines that endotoxemia is observed in both NAFLD and ALD patients. LPS plays an important role in conducting liver damage through the LPS-TLR4 signaling pathway. Treatments targeting the gut microbiome and the gut barrier such as fecal microbiota transplantation (FMT), probiotics, prebiotics, synbiotics, and intestinal alkaline phosphatase (IAP) represent potential treatment modalities for NAFLD and ALD.

Conclusions The gut-liver axis plays an important role in the development of liver disease. Treatments targeting the gut microbiome and the gut barrier have shown beneficial effects in attenuating liver inflammation and need to be further investigated.
\end{abstract}

Keywords NAFLD $\cdot$ ALD $\cdot$ LPS $\cdot$ Gut barrier $\cdot$ Microbiome

\section{Introduction}

Nonalcoholic fatty liver disease (NAFLD) is a pathologic condition defined by the deposition of triglycerides in the liver at greater than $5 \%$ of the total liver weight in the absence of excessive alcohol consumption. ${ }^{1}$ The term NAFLD encompasses a spectrum of pathologic conditions ranging from simple steatosis to nonalcoholic steatohepatitis (NASH), a progressive form of fatty liver disease that may lead to fibrosis, cirrhosis, hepatocellular carcinoma, and death. ${ }^{2}$ NAFLD is now a burgeoning health burden, and it

Florian Kühn

Florian.Kuehn@med.uni-muenchen.de

1 Department of General, Visceral and Transplant Surgery, University Hospital of LMU Munich, Marchionini Str. 15, Munich, Germany is estimated that the prevalence of NAFLD in the general population is $24 \%(20-29 \%){ }^{3}$ Around $20-30 \%$ of NAFLD patients develop NASH, with only some of them further evolving to fibrosis and cirrhosis. ${ }^{4}$ NAFLD carries a large economic burden, contributes to a decreased quality of life, and is becoming a major reason for liver transplantation worldwide. $^{5}$

Alcoholic liver disease (ALD) is a major cause of morbidity and mortality among people who abuse alcohol. ${ }^{6}$ Similar to NAFLD, the spectrum of ALD ranges from simple steatosis to alcoholic steatohepatitis (ASH), fibrosis, cirrhosis, and ultimately hepatocarcinoma. ${ }^{7}$ Alcohol causes $50 \%$ of liver cirrhosis and $10 \%$ of liver cancer related death. ${ }^{8}$ Besides the direct effects of alcohol on liver injury, gut microbiota plays an important role in liver damage. Alcohol intake can lead to changes in gut microbiota composition, even before the onset of liver disease. ${ }^{9}$ 
Lipopolysaccharide (LPS), also known as endotoxin, is a component of the outer cell wall of Gram-negative bacteria. ${ }^{10}$ In humans, the gut microbiota is the major source of LPS. ${ }^{11}$ LPS is generally known for its role in the induction of sepsis, septic shock, and multiple organ failure. ${ }^{12}$ Recently, LPS is found to be related to several other diseases, especially to metabolic disorders such as type 2 diabetes mellitus and atherosclerosis. ${ }^{13,14}$ Furthermore, the role of LPS in neurologic and mental disorders like Alzheimer's disease and autism has been well described, ${ }^{15,16}$ linking gut microbiota to the homeostasis of the entire body.

The connection between gut microbiota and chronic liver disease has been first noticed in the 1980s when NASH was encountered as a common complication of jejunoileal bypass surgery for morbid obesity that could be reversed by treatment with metronidazole. ${ }^{17}$ Bacterial overgrowth in the blind loop has been considered to be responsible for liver damage. Today, the human intestinal microbiota has emerged as an important mediator of the development and progression of chronic liver diseases.

This manuscript briefly reviews the role of gut-derived LPS in the development and progression of NAFLD and ALD. Gut barrier dysfunction and dysbiosis are highlighted as they are the two major mechanisms of endotoxemia as well as potential therapeutic targets.

\section{The Intestinal Barrier and LPS Translocation}

The intestinal barrier serves as a physical and functional barrier deterring translocation of potentially harmful luminal antigens into circulation. The four layers of the intestinal barrier include luminal intestinal alkaline phosphatase (IAP) released from the intestinal epithelial cells, surface mucus, epithelial layer, and immune defense. The epithelial cell layer and the mucin layer constitute the physical barrier; IAP and antibacterial proteins secreted by Paneth cells represent the functional barrier. ${ }^{18}$

IAP dephosphorylates substrates such as LPS and other pathogen-associated molecular patterns. Its role in reducing local intestinal inflammation and maintaining gut barrier function is reviewed later. The intestinal mucosal layer represents the first physical barrier. The small intestine harbors a single, tightly attached mucus layer, whereas in the colon, the mucus is composed of two layers: the essentially sterile inner layer which is firmly attached to the epithelial cells and the outer layer which contains commensal bacteria that prevent the entry of pathogenic bacteria. The epithelium is constituted of a single layer of different intestinal epithelial cells (IECs), and cells within the epithelial layer are sealed by tight junction proteins including claudins, zonula occludens-1 (ZO-1), and occluding, preventing paracellular transport. ${ }^{19}$ The tight junctions (TJs) constitute the major determinant of the intestinal physical barrier and can prevent the paracellular passage of large molecules through the epithelium. ${ }^{20}$

Disruption of the intestinal barrier can cause LPS translocation and cause endotoxemia in systemic circulation and chronic liver inflammation. The permeability of the epithelium is determined by the composition and abundance of different components of the TJs. ${ }^{21}$ Many factors can alter intestinal permeability, such as diet, alcohol intake, medication, and physiological factors such as age and stress. ${ }^{22-24}$ Changes in intestinal microbiota composition can influence intestinal permeability, as a study showing that a high-fat diet changed the gut microbiota content and could increase intestinal permeability. More importantly, this effect was completely restored by antibiotic treatment. ${ }^{25}$ And vice versa, probiotic bacteria and probiotic mixture are shown to have beneficial effects in reestablishing intestinal homeostasis and preserving epithelial barrier function. ${ }^{26}$ Bacterial metabolites, such as short-chain fatty acids (SCFAs), are also reported to play an important role in maintaining both intestinal immune functions and regulating gut barrier functions. ${ }^{27}$

LPS translocate the intestinal barrier mainly through the transcellular pathway, and chylomicrons can also bind and facilitate the absorption of LPS. ${ }^{28}$ Translocation of LPS can occur in physiological states. ${ }^{29}$ Under pathological conditions when there is an increase in gut barrier permeability, paracellular pathway of LPS is increased. The liver is the first organ in the body to encounter gut-derived bacteria and pathogen associated molecular patterns (PAMPs). Chronic exposure to increased levels of PAMPs has been linked to liver diseases. ${ }^{30}$

\section{LPS Conducts Liver Injury Through TLR4 Signaling Pathway}

As the gut is considered to be the first barrier against bacteria, the liver is the second barrier based on the fact that the liver and the gut share both anatomical and functional relations and proximities; their close interaction is also described as the gut-liver axis. ${ }^{31}$ The portal venous system sits at the interface between the host and the inflammatory mediators that exist within the gut. The most important gutderived inflammatory mediator, LPS, enters the liver through the portal vein and is detoxified in the liver. Only few LPS passes through the gut barrier and finally arrives in the liver under physiologic conditions. However, small intestinal bacteria overgrowth (SIBO) or an increase in intestinal permeability leads to translocation of bacteria and its by-products such as LPS. After arriving in the liver, LPS is taken up by hepatocytes and Kupffer cells and is excreted into the bile duct in further process. ${ }^{32}$ In patients with chronic liver 
disease, high LPS portal/peripheral concentrations have been observed. ${ }^{33}$ LPS binds to LPS-binding protein (LBP), and the LBP-LPS complex is transferred to membrane bound or soluble cluster of differentiation 14 (CD14), thereby specifically binds to toll-like receptor 4 (TLR4) and induces the interaction of TLR4 with adaptor molecule myeloid differentiation factor 88 (MyD88). MyD88 further activates downstream mitogen-activated protein kinase (MAPK) and nuclear factor- $\mathrm{kB}$ (NF-kB). Stimulation of the LPS-TLR4 signaling pathway eventually leads to the release of proinflammatory mediators like tumor necrosis factor- $\alpha$ (TNF$\alpha$ ) and interleukin 6 (IL-6). ${ }^{34}$ LPS also mediates signaling through MyD88-independent pathway, but the activation of MAPK and NF-kB occurs in a delayed manner. ${ }^{35}$ In both ALD and NAFLD progression, TLR4 signaling is considered a key pathway, and, very interestingly, it is reported that mice deficient in TLR4 are resistant to both alcohol-induced liver injury and NAFLD. ${ }^{36,37}$

TLR4 is expressed on all types of liver cells, including Kupffer cells, hepatocytes, hepatic stellate cells (HSCs), and also cholangiocytes. Its expression on these cells is correlated with activation of fibrogenic cells and the stage of fibrosis. ${ }^{32}$ In normal liver, hepatic cells express minimal TLRs; therefore, the liver has a high tolerance to TLR ligands. Hepatocytes directly clear LPS as data showed that fluorescence was revealed in hepatocytes 5 min after injection of fluorescein isothiocyanate (FITC)-LPS into the portal vein and then rapidly secreted into the bile. ${ }^{38}$ LPS promotes TNF- $\alpha$ production in Kupffer cells. The inflammatory mediator TNF- $\alpha$ is considered a central mediator in the pathogenesis of both ALD and NAFLD. ${ }^{39}$ Despite the fact that Kupffer cells are the main targets of LPS in the liver, it is the HSCs to promote TLR4-dependent fibrosis. ${ }^{40}$ LPS can activate HSCs in vitro and in vivo, and Kupffer cells strongly enhance this process by producing transforming growth factor beta (TGF- $\beta$ ) and increasing the sensitivity of HSCs to TGF- $\beta$. Recent studies have pointed out that platelets can also play a role in liver injury as biopsy results showed that the number of platelets is associated with disease severity in NAFLD. ${ }^{33}$ Platelets passing through hepatic sinusoids can be activated by LPS and the number of TLR4+ platelets is positively correlated with serum LPS level in NAFLD patients, suggesting that LPS may activate platelets through TLR4 signaling pathway. LPS is capable of activating platelets via TLR4-mediated over-production of eicosanoids, and mice given aspirin showed a decrease of liver fibrosis. ${ }^{41,42}$

\section{Histology of ALD and NAFLD}

ALD shares histological similarities to NAFLD,${ }^{43}$ indicating that there may be a common pathway for liver injury in both diseases. The hallmark of NAFLD and ALD is the excessive fat accumulation in the hepatocytes, which may be an isolated event (simple steatosis) or accompanied by evidence of inflammation and cell injury with or without fibrosis (NASH and ASH) ${ }^{44}$. The histological changes in ALD or NAFLD are lobulocentric (typically affecting zone 3) but not portal-based, ${ }^{45}$ including hepatocyte ballooning, Mallory bodies, zone 3 inflammation, and perisinusoidal fibrosis. Recent studies have identified the importance of portal fibrosis in predicting the subgroup of NAFLD patients that develop progressive liver disease and liver-related mortality. ${ }^{46}$ Portal inflammation is associated with portal-based changes, such as ductular reaction (DR), a reactive lesion at the portal tract interface comprising small biliary ductules with an accompanying complex of stroma and inflammatory cells. ${ }^{47}$ The underlying mechanism of DR is the activation of a secondary proliferative pathway of hepatic progenitor cells (HPCs) during the replacement of necrotic and apoptotic hepatocytes. ${ }^{48}$ Under regular conditions, the primary pathway of liver regeneration is maintained by the replication of adjacent hepatocytes within the lobules. With an insult of toxins, viral infection, alcohol, etc., the primary pathway is blocked and replaced by the secondary pathway: HPCs proliferate and differentiate into hepatocytes and bile ductal epithelia; the by-product is the DR (Figure 1).

Odena et al.$^{49}$ proved that the LPS-TLR4 pathway stimulates the expansion of ductular reaction in alcoholic hepatitis (AH). The study found that the keratin 23 (KRT23) gene, which is expressed in the ductular reaction cells, is the most upregulated gene in AH compared to NASH and normal livers. They discovered that LPS administration markedly induced KRT23 expression in mice. This effect can be attenuated in TLR4-deficient mice, further indicating that the LPS-TLR4 pathway mediates the development of ductular reaction in chronic liver injury.

Another study by Vespasiani-Gentilucci et al. ${ }^{32}$ provided evidence that serum LPS levels correlated with portal/ interface inflammation, the activity of portal/septal myofibroblasts, and fibrosis in NAFLD patients. Using immunohistochemistry, TLR4 expression was observed in hepatic progenitor cells, biliary cells, and portal/septal macrophages. TLR4-positive hepatic progenitor cells and bile ducts/ductules correlate with portal/interface inflammation, activation of fibrogenic cells, and fibrosis, implicating that the LPSTLR4 pathway is associated with inflammation and fibrosis progression in NAFLD.

\section{LPS in the Pathogenesis of NAFLD}

NAFLD is the liver manifestation of metabolic syndrome and is characterized by massive ectopic triglyceride accumulation in the liver in the absence of any other liver disease or significant alcohol consumption. ${ }^{50}$ The etiology of 


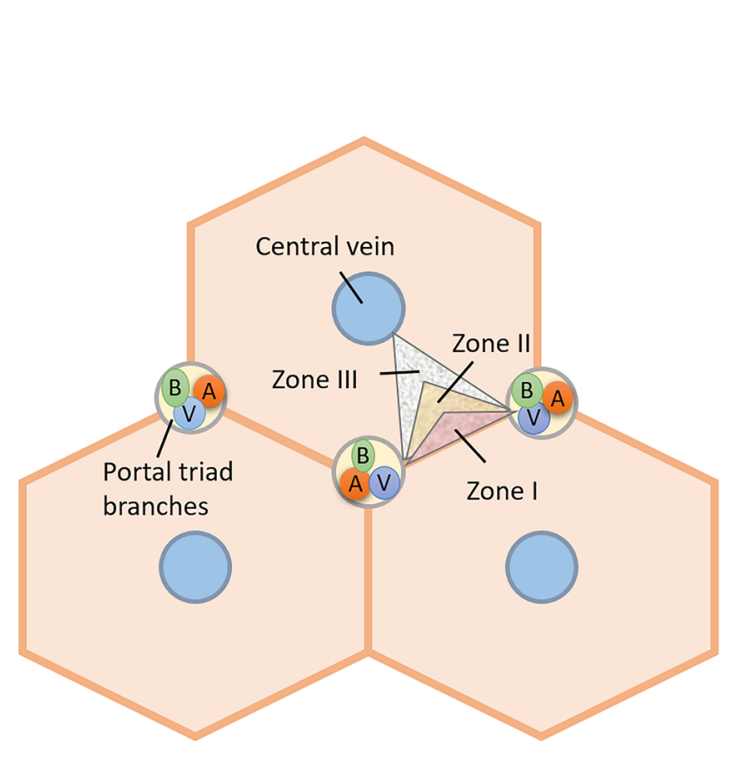

a

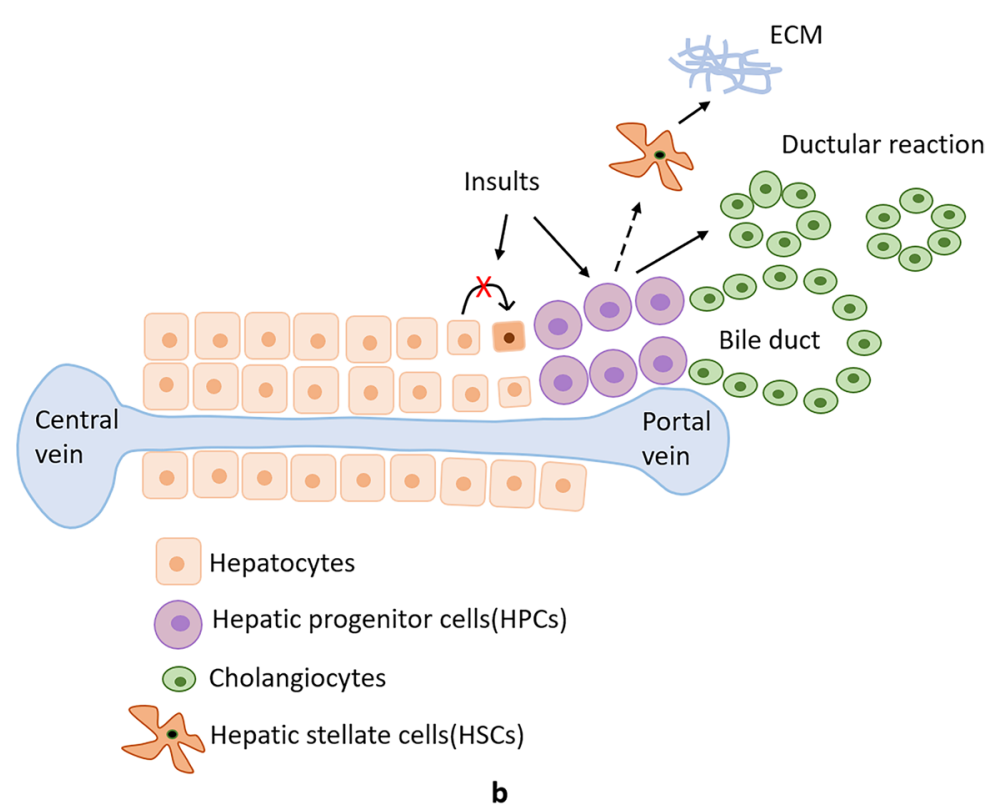

ond pathway of HPCs activation. HPCs are bipotential cells and can differentiate into hepatocytes and cholangiocytes. The latter process causes the ductular reaction (DR). The HPCs can also interact with hepatic stellate cells, which are the primary source of the extracellular matrix (ECM) and the key players of the liver fibrogenic response

liver. ${ }^{56}$ High-fat, high cholesterol diets can result in endotoxemia and low-grade inflammation in both human and animal models. ${ }^{57,58}$ Lifestyle modifications such as diet, physical exercise, and weight loss are advocated. The Mediterranean diet characterized by reduced carbohydrate intake and increased monounsaturated and omega- 3 fatty acid intake has a beneficial effect in overweight patients with NAFLD. ${ }^{59}$ A previous study showed that even short-term diet modification could reduce liver steatosis and steatohepatitis. ${ }^{60}$

Endotoxin plasma level is significantly higher in NAFLD patients, and it seems that endotoxin level is associated with the severity of hepatic steatosis. ${ }^{61}$ Gut dysbiosis is causative for the enhanced secretion of LPS and the resulting inflammation in NAFLD development. ${ }^{62}$ Generally, dysbiosis is defined as an imbalance or alteration in the microbiota that can have an unfavorable effect on the host. ${ }^{63}$ A previous study investigated taxonomic compositions of gut microbiota within the spectrum of NALFD lesions and found that more serious NAFLD lesions (NASH and significant fibrosis) associate with gut dysbiosis. ${ }^{64}$ The study also found that increased levels of Bacteroides were independently associated with NASH and increased Ruminococcus abundance with fibrosis, linking these two taxa of bacteria to the severity of NAFLD.

The gut microbiota composition of an individual is a kind of a fingerprint highly influenced by the type of diet. ${ }^{65} \mathrm{It}$ has been shown that microbiota play an important role in 


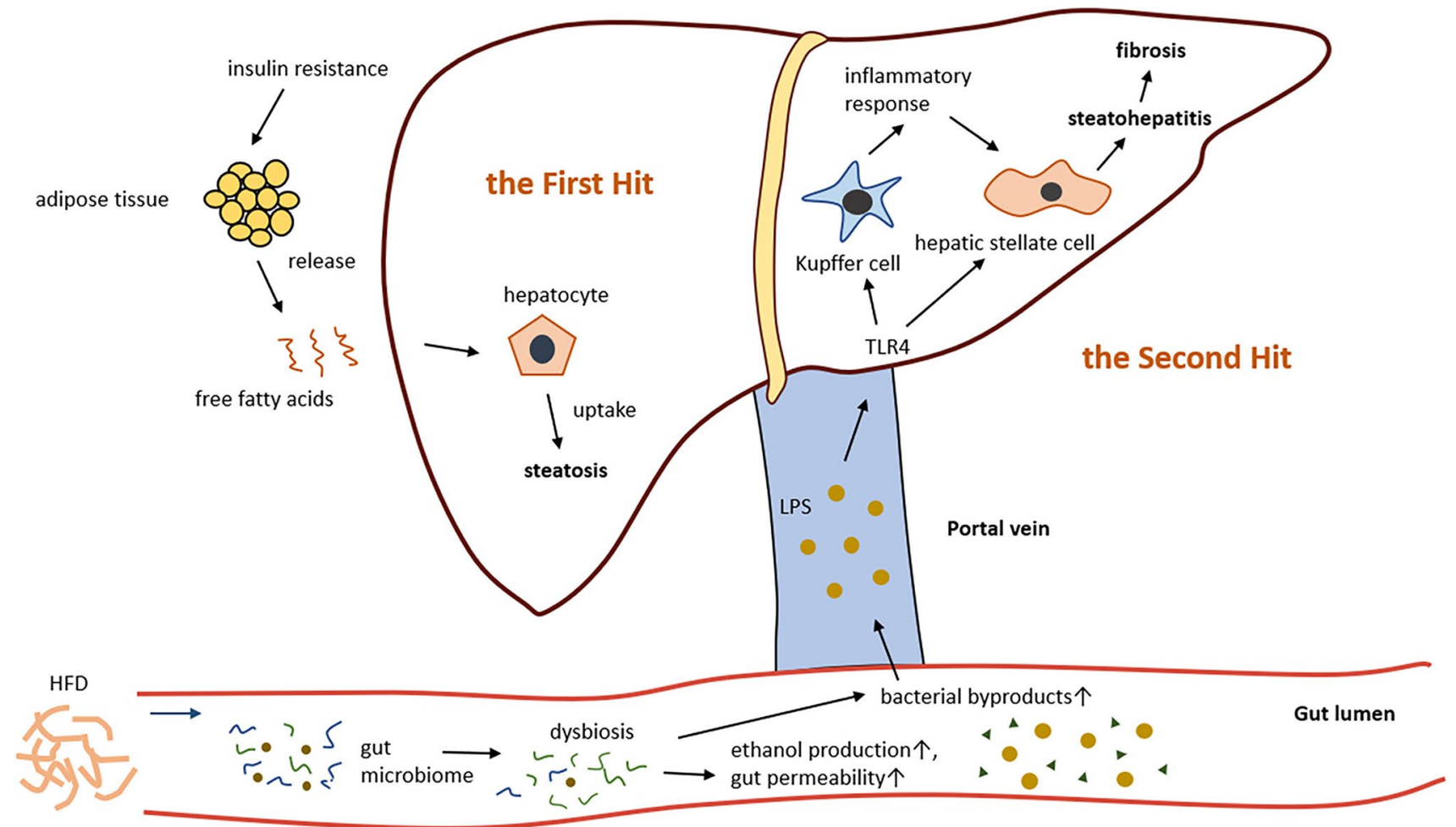

Fig. 2 LPS acts as the second hit in the pathogenesis of NAFLD. Insulin resistance is the major factor for the progression of NAFLD, leading to an increase in free fatty acids (FFAs) in the circulating blood. Excessive uptake of FFAs by hepatocytes results in steatosis, making the liver more vulnerable to further insults, which is considered the "first hit." A high-fat diet (HFD) could lead to gut dysbiosis,

nutrition intake and patients with obesity or metabolic disorder appear to have an intestinal microbiota signature that can harvest more energy than their healthy counterparts. ${ }^{66}$ Changes in abundance and diversity of the gut microbiota in NAFLD have also been characterized. Though many studies have reported conflicting results of the microbiome signatures in NAFLD, an increase in Firmicutes and a reduction in Bacteroidetes is found in most studies. ${ }^{67}$ Thus, a higher Firmicutes/Bacteroidetes ratio is frequently cited as a hallmark of obesity ${ }^{68}$ Furthermore, the amount of ethanol-producing bacteria (e.g., Escherichia coli) was reported to be increased in NAFLD, which may cause increased intestinal permeability and increased translocation of endotoxins from the intestinal lumen to the portal blood. ${ }^{69}$ The impact of microbiota on liver disease is further supported by experiments during which bacterial transfer from the human gut to germ-free (GF) mice resulted in metabolic diseases ${ }^{70,71}$

Guo and collegues ${ }^{72}$ found that LPS per se can cause an increase in intestinal epithelial tight junction permeability in vitro and in vivo. This effect was mediated by an increase in enterocyte TLR4 expression and a TLR4-dependent increase in membrane CD14 expression. Studies have shown that physiologically relevant concentrations of LPS (0 to which further causes an increase in bacterial by-product production and increased gut permeability. Lipopolysaccharide (LPS) translocates the gut barrier, enters the liver through the portal vein, and activates Kupffer cells and hepatic stellate cells (HSCs) through the LPSTLR4 pathway, resulting in an inflammatory response which leads to steatohepatitis and eventually fibrosis

$2000 \mathrm{pg} / \mathrm{mL}$ ) cause an increase in intestinal epithelial TJ permeability. ${ }^{73,74}$ These findings indicate that LPS is an important pathogenic factor in the intestinal inflammatory process.

The gut microbiota is influenced by various factors, including the genetic background of the host, type of diet, age, and medication. How these factors exactly affect gut microbiota composition and function is not fully understood with several studies presenting contradictory data. The regulation of gut microbiota provides a new insight into the treatment of NAFLD.

\section{LPS in the Pathogenesis of ALD}

ALD is associated with high morbidity and mortality rates. Most chronic heavy drinkers develop steatosis, but only $35 \%$ develop advanced liver disease. ${ }^{75}$ A previous study showed that intestinal hyperpermeability occurs only in alcoholics with ALD and not in those without liver disease ${ }^{76}$ Furthermore, chronic alcohol administration has been shown to increase gut-derived endotoxin levels in the portal circulation. ${ }^{77}$ This indicates that alcohol 
plays a crucial role in promoting intestinal hyperpermeability resulting in endotoxemia, systemic inflammation, and liver damage. Alcohol consumption destroys the integrity of the intestinal barrier, disturbs the gut microbiota, and is associated with an increase in the abundance of endotoxin-producing bacterial types. ${ }^{78}$ Metagenomic analysis of the intestinal microbiome of individuals with a history of chronic alcohol abuse has revealed reduced bacterial diversity and a lower proportion of Bacteroidaceae and probiotic bacteria such as Lactobacillus ${ }^{79,80}$. Patients with alcoholic cirrhosis have an increased relative abundance of Enterobacteriaceae. ${ }^{81}$ Alcohol consumption affects bacterial composition within specific phyla. A study by Llopis et al. ${ }^{82}$ found that mice harboring the intestinal microbiota from patients with severe alcoholic hepatitis developed more severe liver inflammation, indicating that individual susceptibility to ALD is substantially driven by the intestinal microbiome.

Both chronic alcohol consumption and acute alcohol intake (binge drinking) can impair the intestinal barrier and increase serum levels of bacterial products. ${ }^{76,83}$ Endotoxemia in ALD was first recognized by the detection of antibodies against Escherichia coli in the plasma of patients with ALD. ${ }^{84}$ Humans and animals with chronic alcohol consumption develop a "leaky gut," as evidenced by higher levels of plasma endotoxin. The plasma endotoxin levels are also associated with the severity of disease. ${ }^{85}$ Alcohol damages specific components of the intestinal barrier such as proteins involved in innate antibacterial defense. Animal experiments have shown that chronic exposure to ethanol can cause gut-barrier disruption featured by a decrease in tight junction protein $\mathrm{ZO}-1$ and occludin expression. ${ }^{86,87}$

Besides ethanol, the by-product acetaldehyde from the metabolization of alcohol by gut microbiota appears to play a crucial role in gut barrier dysfunction. At concentrations ranging from 99 to $760 \mu \mathrm{M}$, acetaldehyde increases the paracellular permeability of Caco- 2 cell monolayer. ${ }^{88}$ The underlying mechanism includes the dissociation of ZO-1 from the junctions in acetaldehydetreated cell monolayer, thus leading to disrupted TJ structure and the increase in paracellular permeability. ${ }^{89}$ The study further revealed that acetaldehyde increases tyrosine phosphorylation of ZO-1, E-cadherin, and $\beta$-catenin, resulting in disruption of the TJs.

Chronic alcohol consumption is also associated with changes in bile acid profiles. ALD patients have an increased secondary bile acid formation. ${ }^{90}$ A study by Xie et al. ${ }^{91}$ showed that ethanol consumption could lead to a substantial decrease in taurine-conjugated (hydrophilic and less toxic) bile acids, resulting in impaired lipid emulsification and liver steatosis in mice.

\section{Intestinal Targeted Therapy}

While simple steatosis is considered a "benign disease," treatment of NAFLD and ALD should focus on NASH and ASH. Currently, there is no targeted and thus effective drug therapy for the treatment of NASH or ASH. Especially, the long-term intake of traditional treatments for chronic liver diseases such as antibiotics and corticosteroids is associated with various side effects for patients. There is accumulating evidence that the interplay between the gut microbiota and the liver is critical in the pathogenesis of chronic liver disease. Hence, there is a large interest in modulating the microbial community to achieve a therapeutic effect or even reverse liver fibrosis. The manipulation of the gut microbiome by fecal microbiota transplantation (FMT), probiotics, prebiotics, and synbiotics was shown to have a beneficial effect in improving liver phenotype in patients with chronic liver disease. IAP as a naturally occurring brush boarder enzyme that detoxifies LPS and preserves microbial homeostasis and gut barrier integrity appears to be a promising candidate for treatment of liver fibrosis in patients with NAFLD or ALD.

\section{Fecal Microbiota Transplantation}

This procedure involves the transfer of processed feces to restore a "healthy microbiome." FMT has been successfully used to treat recurrent Clostridium difficile infection (rCDI) for years ${ }^{92,93}$ and possesses potential in treating gastrointestinal as well as extraintestinal diseases.

FMT has shown an effect in alleviating high-fat dietinduced steatohepatitis in mice. ${ }^{94}$ The therapeutic effect may be caused by an increase in "beneficial" gut microbiota, improving the tight junction of small intestinal and lowering the LPS levels. Ferrere et al. ${ }^{95}$ performed FMT from alcohol-resistant donor mice (alcohol-fed mice did not develop alcohol-induced liver lesions) to alcoholsensitive receiver mice (alcohol-fed mice developed liver lesions) and found that FMT protected the alcohol-sensitive mice from alcohol-induced depletion of Bacteroides. FMT treatment was also found to prevent steatosis in alcohol-fed mice, indicating the beneficial effect of FMT in preventing ALD development. In a small-scale pilot study conducted by Philips et al., ${ }^{96} 8$ patients with steroid-ineligible severe alcoholic hepatitis received FMT treatment from healthy donors. The results showed that indices of liver disease severity, including ascites, hepatic encephalopathy, and mean bilirubin, significantly improved after FMT. FMT treatment also showed an effect on modulation of gut microbiota and improved prognosis in these 
patients. In a recent randomized trial, twenty patients with liver cirrhosis were randomly given FMT capsules or placebo. Patients receiving FMT capsules showed an improved gut microbial function and significantly reduced systemic inflammation markers (IL-6 and LBP). ${ }^{97}$

FMT contributes to restore a balanced gut microbiota composition and has emerged as a therapeutic option for chronic liver disease. Although FMT shows its beneficial effect in animal models and clinical trials of non-alcoholic and alcoholic liver steatosis, it has not been approved for clinical use. Further research is needed to verify the safety and effectiveness of FMT in chronic liver disease.

\section{Probiotics}

Probiotics are defined by the Food and Agriculture Organization (FAO)/World Health Organization (WHO) as living microorganisms that confer a health benefit on the host when administered in adequate amounts. ${ }^{98}$ They exhibit characteristics including tolerance to gastrointestinal conditions, ability to adhere to the gastrointestinal mucosa and competitive exclusion of pathogens. ${ }^{99}$ The strains most frequently used as probiotic bacteria belong to the Bifidobacterium and Lactobacillus genera and are widely used in yogurts and other dairy products. ${ }^{100}$ Probiotics are generally considered to have a beneficial effect on the human intestine. Induction of probiotics may control the growth of pathologic organisms. Studies have proven that probiotics are efficient in treating various diseases such as antibiotic-associated diarrhea (ADD), inflammatory bowel diseases (IBD), and neuropsychiatric disorders. ${ }^{101-103}$

Probiotics have been proven to lower the plasma triglyceride (TG) levels in animal models of metabolic syndrome. ${ }^{104}$ Plaza-Diaz et al. ${ }^{105}$ demonstrated the reduction in liver steatosis of obese mice fed certain probiotic strains. This effect was associated with lower serum LPS levels as matched trends were observed in LPS serum concentration and liver TG content.

Clinical trials have confirmed the beneficial effects of probiotics in nonalcoholic liver diseases both in adults and children. ${ }^{106-108}$ Probiotic treatment has been shown to play a role in improving liver function. The intake of probiotics leads to reduced levels of alanine aminotransferase (ALT), aspartate aminotransferase (AST), glutamine transferase (GGT), total cholesterol (TC), TG, and improved liver histological marker such as NAS (NAFLD Activity Score). ${ }^{107}$ Probiotic supplementation is also able to improve biomarkers of inflammation (e.g., TNF- $\alpha$, IL-6) and steatosis (e.g., arginase, prolidase). ${ }^{109}$

Similar to NAFLD, patients with alcoholic liver disease can also benefit from a probiotic treatment. In a Russian pilot study ${ }^{110}$, Bifidobacterium bifidum and Lactobacillus plantarum $8 P A 3$ were randomly given to patients diagnosed with alcoholic psychosis. The treatment led to the alterations in bowel flora in alcoholic patients and liver enzyme reduction, indicating that probiotics can improve alcoholinduced liver injury. Other randomized-controlled trials of Lactobacillus casei supplements in patients with alcoholic liver injury support this finding. ${ }^{111}$

In general, the mechanisms by which probiotics prevent chronic liver injury include: reducing gut-derived microbial LPS by restoring the bowel flora, repairing the intestinal mucosa and barrier function, modulation of the immune system, and reducing inflammatory cytokine levels. ${ }^{112}$

\section{Prebiotics and Synbiotics}

A prebiotic is a non-viable food component that can confer a health benefit on the host that is based on the modulation of the intestinal microbiota. ${ }^{113}$ Main prebiotics include primarily short- and long-chain fructans (fructo-oligosaccharides (FOS) and inulin), galacto-oligosaccharides (GOS), and lactulose. ${ }^{114}$ These substances possess features including non-digestibility, fermentation by intestinal microflora, and selective stimulation of growth and activity of intestinal bacteria. ${ }^{115}$

Prebiotics have beneficial effects on the gastrointestinal tract, which include prevention of pathogen damage or immune system modulation, improvement of gut barrier function, reduction in the pathogenic bacteria population, and the production of SCFAs. ${ }^{116}$ Prebiotics are used mostly as a selective medium for the growth of a probiotic strain, and Lactobacilli and Bifidobacteria are the usual target genera for prebiotics. Large bundles of studies have shown that prebiotics are able to increase the composition and/or activity of Lactobacillus and Bifidobacterium populations. ${ }^{117-120}$ In lipid metabolism, prebiotics exhibit serum or hepatic lipid-lowering properties, and are now considered a potential dietary adjunct in reducing the risks of cardiovascular diseases (CVD) with minimal side effects ${ }^{121}$. A randomized placebo-controlled trial carried out in Canada showed that, after 16 weeks of consumption of oligofructose-enriched inulin, there was a significant decrease in body weight $z$-score, percent body fat, and percent trunk fat and serum level of IL-6 in healthy children with overweight or obesity compared to the placebo control group. ${ }^{122}$ The study also observed a significantly higher abundance of Bifidobacterium spp. in the prebiotic consumption group, indicating that prebiotic consumption could selectively alter gut microbiota.

The prebiotic inulin is proven to be effective in preventing NAFLD in animal experiments. ${ }^{123,124}$ However, the effect of inulin in human trials remains controversial. Chambers et al. ${ }^{125}$ explored the effects of dietary supplementation with inulin in adults with NAFLD and found that inulin consumed 
at $20 \mathrm{~g} / \mathrm{d}$ increased intrahepatocellular lipid (IHCL). They speculated that the acetate derived from colonic fermentation of inulin could provide an additional lipogenic substrate to the liver.

A synbiotic is a mixture of 1 or more probiotics and 1 or more prebiotics that beneficially affect the host by promoting the survival and colonization of the live microbes in the gastrointestinal tract. ${ }^{115}$ It is known that probiotics are active in the small and large intestine and the effect of a prebiotic is mainly in the colon. The combination of the two may have a synergistic effect. ${ }^{126}$ The intake of synbiotics has been demonstrated to modify the composition of the microbiota, thus protecting against inflammation and hepatocyte damage. ${ }^{127}$ A recent meta-analysis involving 15 randomized clinical trials in which 8 studies about synbiotics were included showed that synbiotics supplementation can improve TC, TG, high-density lipoprotein (HDL), and lowdensity lipoprotein (LDL) in patients with NAFLD, indicating that synbiotics could improve lipid profiles in those patients. ${ }^{128}$ Among these studies, Malaguarnera et al. ${ }^{127}$ conducted a randomized clinical trial in which 66 NASH patients were enrolled and randomly given Bifidobacterium longum with fructo-oligosaccharides or placebo. The results showed that synbiotic treatment improved liver histology in NASH patients and had a beneficial effect in reducing inflammation markers like C-reactive protein (CRP) and TNF- $\alpha$. These effects may be due to reduced LPS exposure to the liver.

Though studies of the relationship between synbiotics and ALD are much lesser, the beneficial effect of synbiotics in attenuating chronic alcohol intake induced liver injury is observed in mice. ${ }^{129,130}$ Synbiotic supplementation was also shown to decrease serum LPS levels in high-risk alcoholic participants and in patients with alcohol-related cirrhosis. ${ }^{131,132}$

\section{Intestinal Alkaline Phosphatase}

IAP is ubiquitously expressed by enterocytes in the proximal small intestine and exists in high concentrations within luminal vesicles secreted by enterocytes on the brush border of the microvilli. ${ }^{133}$ IAP is secreted bilaterally and is also released in small amounts into the blood. ${ }^{134}$ IAP plays an important anti-inflammatory role by dephosphorylating potentially proinflammatory ligands such as adenosine triphosphate (ATP), uridine diphosphate (UDP), unmethylated cytosine-guanosine dinucleotides $(\mathrm{CpG})$, and LPS. ${ }^{135}$ Luminal IAP can prevent and reduce intestinal inflammation and bacterial translocation and is considered the first layer of the intestinal barrier. ${ }^{18}$ The anti-inflammatory effect makes it a potential treatment for various diseases. Previous studies had found decreased levels of IAP protein expression in inflamed colonic mucosa in children with IBD and decreased IAP mRNA levels in inflamed tissue of adults with IBD. ${ }^{136,137}$ Exogenous IAP supplementation showed a beneficial effect in alleviating inflammation in a rat model of IBD. ${ }^{138}$ In a phase II trial, intravenous injection of IAP improved renal function in patients with severe sepsis and septic shock. ${ }^{139}$ And another phase II trial demonstrated that administration of bovine IAP could reduce post-surgical inflammatory response in patients undergoing coronary artery bypass grafting $(\mathrm{CABG}) .{ }^{140}$

IAP is an important enzyme to maintain the integrity of the gut barrier. By dephosphorylating luminal ATP, IAP can act as a component of the ecto-purinergic signaling system to regulate ATP-dependent $\mathrm{HCO}_{3}{ }^{-}$secretion and localized extracellular $\mathrm{pH}\left(\mathrm{pH}_{0}\right) \cdot{ }^{141}$ Liu et al. ${ }^{142}$ demonstrated that IAP can directly regulate TJ protein levels as its gene depletion in mouse embryonic fibroblasts resulted in significantly lower levels of ZO-1, ZO-2, and occludin expression, and IAP overexpression in Caco- 2 and T84 cells resulted in increases in mRNA levels of ZO-1 and ZO-2 (Figure 3). Another study showed a similar result that the intestinal tissue of IAP-KO mice presents a significant decrease in TJ proteins; moreover, oral supplementation with IAP restores TJ protein expression. ${ }^{143}$

IAP also plays a crucial role in regulating the gut microbiota. Malo et al. ${ }^{144}$ examined the status of the gut microbiota in IAP knockout (IAP-KO) mice and found dramatically fewer microbes in their stools compared with wild-type (WT) mice when fed a high-fat diet. Moreover, oral supplementation of calf IAP (cIAP) promoted the restoration of the normal gut microbiota following antibiotic treatment. Kühn et al. ${ }^{145}$ observed the beneficial effect of IAP supplementation in preserving the homeostasis of gut microbiota during aging in mice. IAP has been shown to prevent chronic liver disease in 2 different mice models. Liu et al. ${ }^{146}$ demonstrated that fecal IAP activity decreases in humans with liver cirrhosis and oral supplement of IAP attenuated liver fibrosis in mice. Hamarneh et al. ${ }^{147}$ demonstrated that pretreatment with IAP attenuated the development of alcohol-induced fatty liver, decreased hepatic pro-inflammatory cytokines, as well as serum LPS levels, and prevented alcohol-induced gut barrier dysfunction in mice, indicating that oral IAP supplementation could present a novel therapy to prevent alcoholic-related liver disease. Though the beneficial effect of IAP in chronic liver diseases and maintaining gut barrier function has been observed in various preclinical studies, the therapeutic value of IAP in patients with chronic liver disease is not known.

\section{Conclusion}

Accumulating evidence has pointed out the importance of the gut-liver axis in the development of liver disease. Translocation of bacterial by-products such as LPS results 


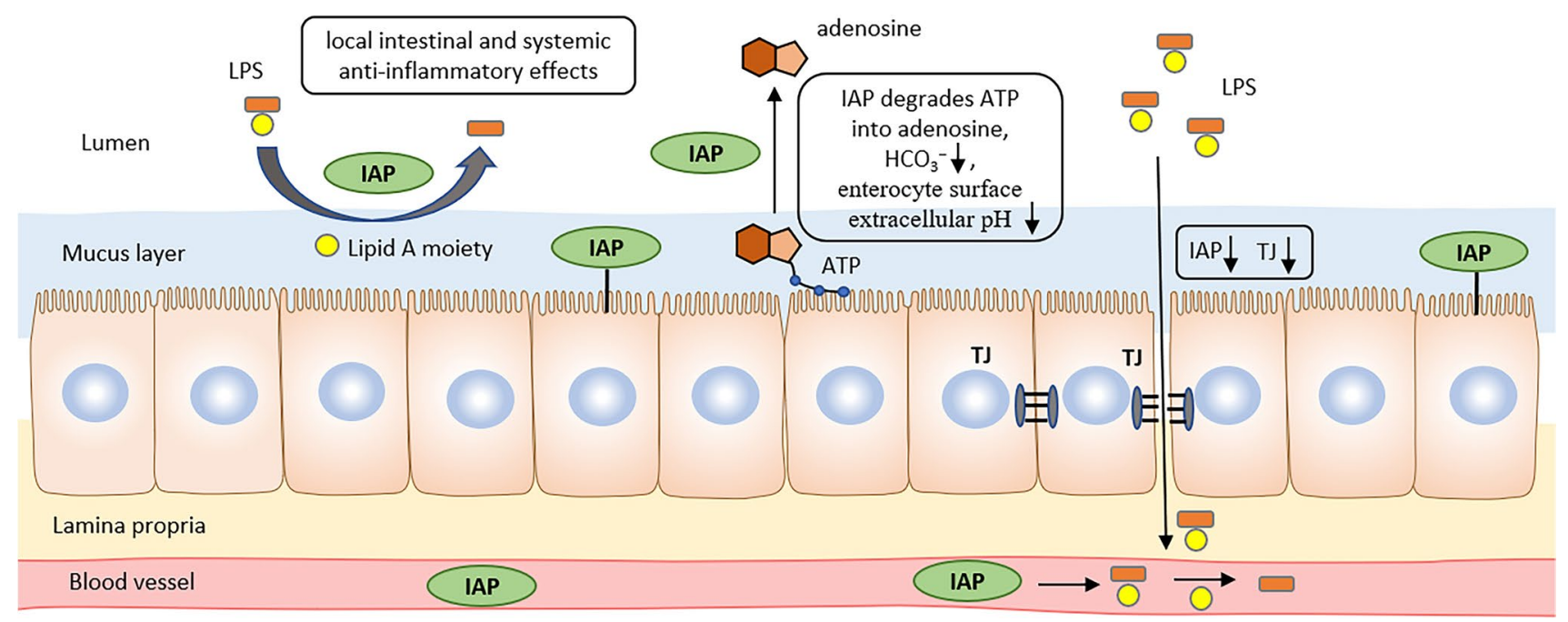

Fig. 3 The role of IAP in preventing local inflammation, preventing LPS translocation, and regulation of enterocyte surface extracellular $\mathrm{pH}$. IAP is highly expressed in the brush border membrane of duodenal epithelial cells and is secreted bilaterally into the gut lumen and the blood. IAP can detoxify LPS, resulting in amelioration of intestinal and systemic inflammation. ATP serves as a substrate for brush

in chronic inflammation in the liver. Treatments targeting the gut microbiome such as FMT, probiotics, prebiotics, and synbiotics have shown an effect in attenuating liver inflammation. IAP possessing an anti-inflammatory effect by dephosphorylating LPS and maintaining gut barrier function is also showing therapeutic potential. Current evidence demonstrates that maintaining gut barrier integrity and microbiome homeostasis is of great significance in treating liver diseases.

Author Contribution All authors have contributed substantially to the conception of this review and devised or revised the manuscript for intellectual content and have approved the final version for publication. In addition, all authors agree to be accountable for all aspects of the work.

Funding Open Access funding enabled and organized by Projekt DEAL.

\section{Declarations}

Conflict of Interests The authors declare no competing interests.

Open Access This article is licensed under a Creative Commons Attribution 4.0 International License, which permits use, sharing, adaptation, distribution and reproduction in any medium or format, as long as you give appropriate credit to the original author(s) and the source, provide a link to the Creative Commons licence, and indicate if changes were made. The images or other third party material in this article are included in the article's Creative Commons licence, unless indicated otherwise in a credit line to the material. If material is not included in the article's Creative Commons licence and your intended use is not permitted by statutory regulation or exceeds the permitted use, you will need to obtain permission directly from the copyright holder. To view a copy of this licence, visit http://creativecommons.org/licenses/by/4.0/. border IAP. In the gut lumen, the presence of ATP increases HCO3secretion. IAP decreases luminal ATP concentration and diminish this pathway. IAP also plays a role in regulating tight junction protein levels, preserving gut integrity and preventing translocation of bacterial by-products

\section{References}

1. Chalasani N, Younossi Z, Lavine JE, et al. The diagnosis and management of nonalcoholic fatty liver disease: Practice guidance from the American Association for the Study of Liver Diseases. Hepatology. 2018;67(1):328-357.

2. Calzadilla Bertot L, Adams LA. The Natural Course of NonAlcoholic Fatty Liver Disease. Int J Mol Sci. 2016;17(5):774.

3. Younossi ZM, Koenig AB, Abdelatif D, Fazel Y, Henry L, Wymer M. Global epidemiology of nonalcoholic fatty liver disease-Meta-analytic assessment of prevalence, incidence, and outcomes. Hepatology. 2016;64(1):73-84.

4. Singh S, Allen AM, Wang Z, Prokop LJ, Murad MH, Loomba $\mathrm{R}$. Fibrosis progression in nonalcoholic fatty liver vs nonalcoholic steatohepatitis: a systematic review and meta-analysis of paired-biopsy studies. Clin Gastroenterol Hepatol. 2015;13(4):643-654 e641-649; quiz e639-640.

5. Sherif ZA, Saeed A, Ghavimi S, et al. Global Epidemiology of Nonalcoholic Fatty Liver Disease and Perspectives on US Minority Populations. Dig Dis Sci. 2016;61(5):1214-1225.

6. Singal AK, Bataller R, Ahn J, Kamath PS, Shah VH. ACG Clinical Guideline: Alcoholic Liver Disease. Am J Gastroenterol. 2018;113(2):175-194.

7. Beier JI, McClain CJ. Mechanisms and cell signaling in alcoholic liver disease. Biol Chem. 2010;391(11):1249-1264.

8. Avila MA, Dufour JF, Gerbes AL, et al. Recent advances in alcohol-related liver disease (ALD): summary of a Gut round table meeting. Gut. 2020;69(4):764-780.

9. Bajaj JS. Alcohol, liver disease and the gut microbiota. Nat Rev Gastroenterol Hepatol. 2019;16(4):235-246.

10. Tuin A, Huizinga-Van der Vlag A, van Loenen-Weemaes AM, Meijer DK, Poelstra K. On the role and fate of LPS-dephosphorylating activity in the rat liver. Am J Physiol Gastrointest Liver Physiol. 2006;290(2):G377-385.

11. Berg RD. The indigenous gastrointestinal microflora. Trends Microbiol. 1996;4(11):430-435. 
12. Opal SM. Endotoxins and other sepsis triggers. Contrib Nephrol. 2010;167:14-24.

13. Gomes JMG, Costa JA, Alfenas RCG. Metabolic endotoxemia and diabetes mellitus: A systematic review. Metabolism. 2017;68:133-144.

14. Verhaar BJH, Prodan A, Nieuwdorp M, Muller M. Gut Microbiota in Hypertension and Atherosclerosis: A Review. Nutrients. 2020;12(10).

15. Zhan X, Stamova B, Sharp FR. Lipopolysaccharide Associates with Amyloid Plaques, Neurons and Oligodendrocytes in Alzheimer's Disease Brain: A Review. Front Aging Neurosci. 2018;10:42.

16. Srikantha P, Mohajeri MH. The Possible Role of the Microbiota-Gut-Brain-Axis in Autism Spectrum Disorder. Int J Mol Sci. 2019;20(9):2115.

17. Drenick EJ, Fisler J, Johnson D. Hepatic steatosis after intestinal bypass--prevention and reversal by metronidazole, irrespective of protein-calorie malnutrition. Gastroenterology. 1982;82(3):535-548.

18. Ghosh S, Yannie PJ, Wang J, Ghosh SS. Intestinal Barrier Dysfunction, LPS Translocation, and Disease Development. Journal of the Endocrine Society. 2020;4(2):bvz039.

19. Vancamelbeke M, Vermeire S. The intestinal barrier: a fundamental role in health and disease. Expert Rev Gastroenterol Hepatol. 2017;11(9):821-834.

20. Forster $\mathrm{C}$. Tight junctions and the modulation of barrier function in disease. Histochem Cell Biol. 2008;130(1):55-70.

21. Wells JM, Brummer RJ, Derrien M, et al. Homeostasis of the gut barrier and potential biomarkers. Am J Physiol Gastrointest Liver Physiol. 2017;312(3):G171-G193.

22. Mullin JM, Valenzano MC, Verrecchio JJ, Kothari R. Age- and diet-related increase in transepithelial colon permeability of Fischer 344 rats. Dig Dis Sci. 2002;47(10):2262-2270.

23. Karl JP, Margolis LM, Madslien EH, et al. Changes in intestinal microbiota composition and metabolism coincide with increased intestinal permeability in young adults under prolonged physiological stress. Am J Physiol Gastrointest Liver Physiol. 2017;312(6):G559-G571.

24. Jennison E, Byrne CD. The role of the gut microbiome and diet in the pathogenesis of non-alcoholic fatty liver disease. Clin Mol Hepatol. 2021;27(1):22-43.

25. Cani PD, Bibiloni R, Knauf C, et al. Changes in gut microbiota control metabolic endotoxemia-induced inflammation in high-fat diet-induced obesity and diabetes in mice. Diabetes. 2008;57(6):1470-1481.

26. Otte JM, Podolsky DK. Functional modulation of enterocytes by gram-positive and gram-negative microorganisms. Am J Physiol Gastrointest Liver Physiol. 2004;286(4):G613-626.

27. Suzuki T. Regulation of intestinal epithelial permeability by tight junctions. Cell Mol Life Sci. 2013;70(4):631-659.

28. Guerville M, Boudry G. Gastrointestinal and hepatic mechanisms limiting entry and dissemination of lipopolysaccharide into the systemic circulation. Am J Physiol Gastrointest Liver Physiol. 2016;311(1):G1-G15.

29. Clark E, Hoare C, Tanianis-Hughes J, Carlson GL, Warhurst G. Interferon gamma induces translocation of commensal Escherichia coli across gut epithelial cells via a lipid raft-mediated process. Gastroenterology. 2005;128(5):1258-1267.

30. Wang L, Llorente C, Hartmann P, Yang AM, Chen P, Schnabl B. Methods to determine intestinal permeability and bacterial translocation during liver disease. J Immunol Methods. 2015;421:44-53.

31. Malaguarnera G. Gut microbiota in alcoholic liver disease: Pathogenetic role and therapeutic perspectives. World Journal of Gastroenterology. 2014;20(44):16639.
32. Vespasiani-Gentilucci U, Carotti S, Perrone G, et al. Hepatic toll-like receptor 4 expression is associated with portal inflammation and fibrosis in patients with NAFLD. Liver Int. 2015;35(2):569-581.

33. Carpino G, Del Ben M, Pastori D, et al. Increased Liver Localization of Lipopolysaccharides in Human and Experimental NAFLD. Hepatology. 2020;72(2):470-485.

34. Roh YS, Seki E. Toll-like receptors in alcoholic liver disease, non-alcoholic steatohepatitis and carcinogenesis. J Gastroenterol Hepatol. 2013;28 Suppl 1:38-42.

35. Lu YC, Yeh WC, Ohashi PS. LPS/TLR4 signal transduction pathway. Cytokine. 2008;42(2):145-151.

36. Liu J, Zhuang ZJ, Bian DX, et al. Toll-like receptor-4 signalling in the progression of non-alcoholic fatty liver disease induced by high-fat and high-fructose diet in mice. Clin Exp Pharmacol Physiol. 2014;41(7):482-488.

37. Uesugi T, Froh M, Arteel GE, Bradford BU, Thurman RG. Tolllike receptor 4 is involved in the mechanism of early alcoholinduced liver injury in mice. Hepatology. 2001;34(1):101-108.

38. Mimura Y, Sakisaka S, Harada M, Sata M, Tanikawa K. Role of hepatocytes in direct clearance of lipopolysaccharide in rats. Gastroenterology. 1995;109(6):1969-1976.

39. Kawaratani H, Tsujimoto T, Douhara A, et al. The effect of inflammatory cytokines in alcoholic liver disease. Mediators Inflamm. 2013;2013:495156.

40. Seki E, De Minicis S, Osterreicher $\mathrm{CH}$, et al. TLR4 enhances TGF-beta signaling and hepatic fibrosis. Nat Med. 2007;13(11):1324-1332.

41. Li CJ, Yang ZH, Shi XL, Liu DL. Effects of aspirin and enoxaparin in a rat model of liver fibrosis. World J Gastroenterol. 2017;23(35):6412-6419.

42. Nocella C, Carnevale R, Bartimoccia S, et al. Lipopolysaccharide as trigger of platelet aggregation via eicosanoid over-production. Thromb Haemost. 2017;117(8):1558-1570.

43. Rakha EA, Adamson L, Bell E, et al. Portal inflammation is associated with advanced histological changes in alcoholic and nonalcoholic fatty liver disease. J Clin Pathol. 2010;63(9):790-795.

44. Higuera-de la Tijera F, Servin-Caamano AI. Pathophysiological mechanisms involved in non-alcoholic steatohepatitis and novel potential therapeutic targets. World J Hepatol. 2015;7(10):1297-1301.

45. Brunt EM, Janney CG, Di Bisceglie AM, Neuschwander-Tetri BA, Bacon BR. Nonalcoholic steatohepatitis: a proposal for grading and staging the histological lesions. Am J Gastroenterol. 1999;94(9):2467-2474.

46. Younossi ZM, Stepanova M, Rafiq N, et al. Pathologic criteria for nonalcoholic steatohepatitis: interprotocol agreement and ability to predict liver-related mortality. Hepatology. 2011;53(6):1874-1882.

47. Gouw AS, Clouston AD, Theise ND. Ductular reactions in human liver: diversity at the interface. Hepatology. 2011;54(5):1853-1863.

48. Bria A, Marda J, Zhou J, et al. Hepatic progenitor cell activation in liver repair. Liver Res. 2017;1(2):81-87.

49. Odena G, Chen J, Lozano JJ, et al. LPS-TLR4 Pathway Mediates Ductular Cell Expansion in Alcoholic Hepatitis. Sci Rep. 2016;6:35610.

50. Ekstedt M, Nasr P, Kechagias S. Natural History of NAFLD/ NASH. Curr Hepatol Rep. 2017;16(4):391-397.

51. Day CP, James OF. Steatohepatitis: a tale of two "hits"? Gastroenterology. 1998;114(4):842-845.

52. Tilg H, Moschen AR. Evolution of inflammation in nonalcoholic fatty liver disease: the multiple parallel hits hypothesis. Hepatology. 2010;52(5):1836-1846. 
53. Adams LA, Lymp JF, St Sauver J, et al. The natural history of nonalcoholic fatty liver disease: a population-based cohort study. Gastroenterology. 2005;129(1):113-121.

54. Vernon G, Baranova A, Younossi ZM. Systematic review: the epidemiology and natural history of non-alcoholic fatty liver disease and non-alcoholic steatohepatitis in adults. Aliment Pharmacol Ther. 2011;34(3):274-285.

55. Tilg H, Moschen AR, Roden M. NAFLD and diabetes mellitus. Nat Rev Gastroenterol Hepatol. 2017;14(1):32-42.

56. Qureshi K, Abrams GA. Metabolic liver disease of obesity and role of adipose tissue in the pathogenesis of nonalcoholic fatty liver disease. World J Gastroenterol. 2007;13(26):3540-3553.

57. Cani PD, Amar J, Iglesias MA, et al. Metabolic endotoxemia initiates obesity and insulin resistance. Diabetes. 2007;56(7):1761-1772.

58. Erridge C, Attina T, Spickett CM, Webb DJ. A high-fat meal induces low-grade endotoxemia: evidence of a novel mechanism of postprandial inflammation. Am J Clin Nutr. 2007;86(5):1286-1292.

59. Romero-Gomez M, Zelber-Sagi S, Trenell M. Treatment of NAFLD with diet, physical activity and exercise. $J$ Hepatol. 2017;67(4):829-846.

60. Reeves JG, Suriawinata AA, Ng DP, Holubar SD, Mills JB, Barth $\mathrm{RJ}$, Jr. Short-term preoperative diet modification reduces steatosis and blood loss in patients undergoing liver resection. Surgery. 2013; 154(5):1031-1037.

61. Nier A, Huber Y, Labenz C, Michel M, Bergheim I, Schattenberg JM. Adipokines and Endotoxemia Correlate with Hepatic Steatosis in Non-Alcoholic Fatty Liver Disease (NAFLD). Nutrients. 2020;12(3):699.

62. Saltzman ET, Palacios T, Thomsen M, Vitetta L. Intestinal Microbiome Shifts, Dysbiosis, Inflammation, and Non-alcoholic Fatty Liver Disease. Front Microbiol. 2018;9:61.

63. DeGruttola AK, Low D, Mizoguchi A, Mizoguchi E. Current Understanding of Dysbiosis in Disease in Human and Animal Models. Inflamm Bowel Dis. 2016;22(5):1137-1150.

64. Boursier J, Mueller O, Barret M, et al. The severity of nonalcoholic fatty liver disease is associated with gut dysbiosis and shift in the metabolic function of the gut microbiota. Hepatology. 2016;63(3):764-775.

65. Lambertz J, Weiskirchen S, Landert S, Weiskirchen R. Fructose: A Dietary Sugar in Crosstalk with Microbiota Contributing to the Development and Progression of Non-Alcoholic Liver Disease. Front Immunol. 2017;8:1159.

66. Cani PD, Delzenne NM. The role of the gut microbiota in energy metabolism and metabolic disease. Curr Pharm Des. 2009; 15(13):1546-1558.

67. Nier A, Huber Y, Labenz C, Michel M, Bergheim I, Schattenberg JM. Adipokines and Endotoxemia Correlate with Hepatic Steatosis in Non-Alcoholic Fatty Liver Disease (NAFLD). Nutrients. 2020;12(3):699.

68. Magne F, Gotteland M, Gauthier L, et al. The Firmicutes/Bacteroidetes Ratio: A Relevant Marker of Gut Dysbiosis in Obese Patients? Nutrients. 2020;12(5):1474.

69. Zhu L, Baker SS, Gill C, et al. Characterization of gut microbiomes in nonalcoholic steatohepatitis (NASH) patients: a connection between endogenous alcohol and NASH. Hepatology. 2013;57(2):601-609.

70. Fei N, Bruneau A, Zhang X, et al. Endotoxin Producers Overgrowing in Human Gut Microbiota as the Causative Agents for Nonalcoholic Fatty Liver Disease. mBio. 2020;11(1):e03263-19.

71. Chiu CC, Ching YH, Li YP, et al. Nonalcoholic Fatty Liver Disease Is Exacerbated in High Fat Diet-Fed Gnotobiotic Mice by Colonization with the Gut Microbiota from Patients with Nonalcoholic Steatohepatitis. Nutrients. 2017;9(11):1220
72. Guo S, Al-Sadi R, Said HM, Ma TY. Lipopolysaccharide causes an increase in intestinal tight junction permeability in vitro and in vivo by inducing enterocyte membrane expression and localization of TLR-4 and CD14. Am J Pathol. 2013;182(2):375-387.

73. Chen SW, Wang PY, Zhu J, et al. Protective effect of 1,25-dihydroxyvitamin $\mathrm{d} 3$ on lipopolysaccharide-induced intestinal epithelial tight junction injury in caco-2 cell monolayers. Inflammation. 2015;38(1):375-383.

74. Nighot M, Rawat M, Al-Sadi R, Castillo EF, Nighot P, Ma TY. Lipopolysaccharide-Induced Increase in Intestinal Permeability Is Mediated by TAK-1 Activation of IKK and MLCK/MYLK Gene. Am J Pathol. 2019;189(4):797-812.

75. Osna NA, Donohue TM, Jr., Kharbanda KK. Alcoholic Liver Disease: Pathogenesis and Current Management. Alcohol Res. 2017;38(2):147-161.

76. Keshavarzian A, Holmes EW, Patel M, Iber F, Fields JZ, Pethkar S. Leaky gut in alcoholic cirrhosis: a possible mechanism for alcohol-induced liver damage. Am J Gastroenterol. 1999;94(1):200-207.

77. Nanji AA, Khettry U, Sadrzadeh SM, Yamanaka T. Severity of liver injury in experimental alcoholic liver disease. Correlation with plasma endotoxin, prostaglandin E2, leukotriene B4, and thromboxane B2. Am J Pathol. 1993;142(2):367-373.

78. Bajaj JS, Heuman DM, Hylemon PB, et al. Altered profile of human gut microbiome is associated with cirrhosis and its complications. J Hepatol. 2014;60(5):940-947.

79. Gurwara S, Dai A, Ajami NJ, et al. Alcohol use alters the colonic mucosa-associated gut microbiota in humans. Nutr Res. 2020;83:119-128.

80. Yan AW, Fouts DE, Brandl J, et al. Enteric dysbiosis associated with a mouse model of alcoholic liver disease. Hepatology. 2011;53(1):96-105.

81. Lachar J, Bajaj JS. Changes in the Microbiome in Cirrhosis and Relationship to Complications: Hepatic Encephalopathy, Spontaneous Bacterial Peritonitis, and Sepsis. Semin Liver Dis. 2016;36(4):327-330.

82. Llopis M, Cassard AM, Wrzosek L, et al. Intestinal microbiota contributes to individual susceptibility to alcoholic liver disease. Gut. 2016;65(5):830-839.

83. Bala S, Marcos M, Gattu A, Catalano D, Szabo G. Acute binge drinking increases serum endotoxin and bacterial DNA levels in healthy individuals. PLoS One. 2014;9(5):e96864.

84. Staun-Olsen P, Bjorneboe M, Prytz H, Thomsen AC, Orskov F. Escherichia coli antibodies in alcoholic liver disease. Correlation to alcohol consumption, alcoholic hepatitis, and serum IgA. Scand J Gastroenterol. 1983;18(7):889-896.

85. Fujimoto M, Uemura M, Nakatani Y, et al. Plasma endotoxin and serum cytokine levels in patients with alcoholic hepatitis: relation to severity of liver disturbance. Alcohol Clin Exp Res. 2000;24(4 Suppl):48S-54S.

86. Gimenez-Gomez P, Perez-Hernandez M, O'Shea E, et al. Changes in brain kynurenine levels via gut microbiota and gut-barrier disruption induced by chronic ethanol exposure in mice. FASEB J. 2019;33(11):12900-12914.

87. Tang Y, Banan A, Forsyth CB, et al. Effect of alcohol on miR-212 expression in intestinal epithelial cells and its potential role in alcoholic liver disease. Alcohol Clin Exp Res. 2008;32(2):355-364.

88. Rao RK. Acetaldehyde-induced increase in paracellular permeability in Caco-2 cell monolayer. Alcohol Clin Exp Res. 1998;22(8):1724-1730.

89. Atkinson KJ, Rao RK. Role of protein tyrosine phosphorylation in acetaldehyde-induced disruption of epithelial tight junctions. Am J Physiol Gastrointest Liver Physiol. 2001;280(6):G1280-1288. 
90. Kakiyama G, Hylemon PB, Zhou H, et al. Colonic inflammation and secondary bile acids in alcoholic cirrhosis. Am J Physiol Gastrointest Liver Physiol. 2014;306(11):G929-937.

91. Xie G, Zhong W, Li H, et al. Alteration of bile acid metabolism in the rat induced by chronic ethanol consumption. FASEB J. 2013;27(9):3583-3593.

92. Eiseman B, Silen W, Bascom GS, Kauvar AJ. Fecal enema as an adjunct in the treatment of pseudomembranous enterocolitis. Surgery. 1958;44(5):854-859.

93. Kelly CR, Khoruts A, Staley C, et al. Effect of Fecal Microbiota Transplantation on Recurrence in Multiply Recurrent Clostridium difficile Infection: A Randomized Trial. Ann Intern Med. 2016;165(9):609-616.

94. Zhou D, Pan Q, Shen F, et al. Total fecal microbiota transplantation alleviates high-fat diet-induced steatohepatitis in mice via beneficial regulation of gut microbiota. Sci Rep. 2017;7(1):1529.

95. Ferrere G, Wrzosek L, Cailleux F, et al. Fecal microbiota manipulation prevents dysbiosis and alcohol-induced liver injury in mice. J Hepatol. 2017;66(4):806-815.

96. Philips CA, Pande A, Shasthry SM, et al. Healthy Donor Fecal Microbiota Transplantation in Steroid-Ineligible Severe Alcoholic Hepatitis: A Pilot Study. Clin Gastroenterol Hepatol. 2017;15(4):600-602

97. 97.Meighani A, Alimirah M, Ramesh M, Salgia R. Fecal Microbiota Transplantation for Clostridioides Difficile Infection in Patients with Chronic Liver Disease. Int J Hepatol. 2020;2020:1874570.

98. Indian Council of Medical Research Task F, Co-ordinating Unit I, Co-ordinating Unit DBT. ICMR-DBT guidelines for evaluation of probiotics in food. Indian J Med Res. 2011;134:22-25.

99. Zielinska D, Kolozyn-Krajewska D. Food-Origin Lactic Acid Bacteria May Exhibit Probiotic Properties: Review. Biomed Res Int. 2018;2018:5063185.

100. Gourbeyre P, Denery S, Bodinier M. Probiotics, prebiotics, and synbiotics: impact on the gut immune system and allergic reactions. J Leukoc Biol. 2011;89(5):685-695.

101. McFarland LV. Meta-analysis of probiotics for the prevention of antibiotic associated diarrhea and the treatment of Clostridium difficile disease. Am J Gastroenterol. 2006;101(4):812-822.

102. Hedin C, Whelan K, Lindsay JO. Evidence for the use of probiotics and prebiotics in inflammatory bowel disease: a review of clinical trials. Proc Nutr Soc. 2007;66(3):307-315.

103. Morkl S, Butler MI, Holl A, Cryan JF, Dinan TG. Probiotics and the Microbiota-Gut-Brain Axis: Focus on Psychiatry. Curr Nutr Rep. 2020;9(3):171-182.

104. Park DY, Ahn YT, Huh CS, McGregor RA, Choi MS. Dual probiotic strains suppress high fructose-induced metabolic syndrome. World J Gastroenterol. 2013;19(2):274-283.

105. Plaza-Diaz J, Gomez-Llorente C, Abadia-Molina F, et al. Effects of Lactobacillus paracasei CNCM I-4034, Bifidobacterium breve CNCM I-4035 and Lactobacillus rhamnosus CNCM I-4036 on hepatic steatosis in Zucker rats. PLoS One. 2014;9(5):e98401

106. Wang W, Shi LP, Shi L, Xu L. [Efficacy of probiotics on the treatment of non-alcoholic fatty liver disease]. Zhonghua Nei Ke Za Zhi. 2018;57(2):101-106.

107. Cai GS, Su H, Zhang J. Protective effect of probiotics in patients with non-alcoholic fatty liver disease. Medicine (Baltimore). 2020;99(32):e21464.

108. Famouri F, Shariat Z, Hashemipour M, Keikha M, Kelishadi R. Effects of Probiotics on Nonalcoholic Fatty Liver Disease in Obese Children and Adolescents. J Pediatr Gastroenterol Nutr. 2017;64(3):413-417.

109. Tenorio-Jimenez C, Martinez-Ramirez MJ, Tercero-Lozano M, et al. Evaluation of the effect of Lactobacillus reuteri V3401 on biomarkers of inflammation, cardiovascular risk and liver steatosis in obese adults with metabolic syndrome: a randomized clinical trial (PROSIR). BMC Complement Altern Med. 2018;18(1):306

110. Kirpich IA, Solovieva NV, Leikhter SN, et al. Probiotics restore bowel flora and improve liver enzymes in human alcohol-induced liver injury: a pilot study. Alcohol. 2008;42(8):675-682.

111. Li X, Liu Y, Guo X, Ma Y, Zhang H, Liang H. Effect of Lactobacillus casei on lipid metabolism and intestinal.microflora in patients with alcoholic liver injury. Eur J Clin Nutr. 2021;75(8):1227-1236. https://doi.org/10.1007/s11605-021-0518

112. Butel MJ. Probiotics, gut microbiota and health. Med Mal Infect. 2014;44(1):1-8.

113. Pineiro M, Asp NG, Reid G, et al. FAO Technical meeting on prebiotics. J Clin Gastroenterol. 2008;42 Suppl 3 Pt 2:S156-159.

114. Guarino MPL, Altomare A, Emerenziani S, et al. Mechanisms of Action of Prebiotics and Their Effects on Gastro-Intestinal Disorders in Adults. Nutrients. 2020;12(4):1037.

115. Kolida S, Gibson GR. Synbiotics in health and disease. Annu Rev Food Sci Technol. 2011;2:373-393.

116. Slavin J. Fiber and prebiotics: mechanisms and health benefits. Nutrients. 2013;5(4):1417-1435.

117. Bindels LB, Delzenne NM, Cani PD, Walter J. Towards a more comprehensive concept for prebiotics. Nat Rev Gastroenterol Hepatol. 2015;12(5):303-310.

118. Costabile A, Deaville ER, Morales AM, Gibson GR. Prebiotic Potential of a Maize-Based Soluble Fibre and Impact of Dose on the Human Gut Microbiota. PLoS One. 2016;11(1):e0144457.

119. Ramnani P, Costabile A, Bustillo AG, Gibson GR. A randomised, double- blind, cross-over study investigating the prebiotic effect of agave fructans in healthy human subjects. J Nutr Sci. 2015;4:e10.

120. Tandon D, Haque MM, Gote M, et al. A prospective randomized, double-blind, placebo-controlled, dose-response relationship study to investigate efficacy of fructo-oligosaccharides (FOS) on human gut microflora. Sci Rep. 2019;9(1):5473.

121. Olas B. Probiotics, Prebiotics and Synbiotics-A Promising Strategy in Prevention and Treatment of Cardiovascular Diseases? Int J Mol Sci. 2020;21(24):9737.

122. Nicolucci AC, Hume MP, Martinez I, Mayengbam S, Walter J, Reimer RA. Prebiotics Reduce Body Fat and Alter Intestinal Microbiota in Children Who Are Overweight or With Obesity. Gastroenterology. 2017;153(3):711-722.

123. Bao T, He F, Zhang X, et al. Inulin Exerts Beneficial Effects on Non-Alcoholic Fatty Liver Disease via Modulating gut Microbiome and Suppressing the Lipopolysaccharide-Toll-Like Receptor 4-Mpsi-Nuclear Factor-kappaB-Nod-Like Receptor Protein 3 Pathway via gut-Liver Axis in Mice. Front Pharmacol. 2020;11:558525.

124. Bao T, Wang Z, Zhu L, et al. [Inulin increases the proportion of monocytic myeloid-derived suppressor cells in peripheral blood, liver, spleen and regulates the secretion of plasma inflammatory cytokines in mice with non-alcoholic fatty liver disease]. Xi Bao Yu Fen Zi Mian Yi Xue Za Zhi. 2020;36(3):228-235.

125. Chambers ES, Byrne CS, Rugyendo A, et al. The effects of dietary supplementation with inulin and inulin-propionate ester on hepatic steatosis in adults with non-alcoholic fatty liver disease. Diabetes Obes Metab. 2019;21(2):372-376.

126. Macfarlane GT, Cummings JH. Probiotics and prebiotics: can regulating the activities of intestinal bacteria benefit health? BMJ. 1999;318(7189):999-1003.

127. Malaguarnera M, Greco F, Barone G, Gargante MP, Malaguarnera M, Toscano MA. Bifidobacterium longum with fructo-oligosaccharide (FOS) treatment in minimal hepatic encephalopathy: a randomized, double-blind, placebo-controlled study. Dig Dis Sci. 2007;52(11):3259-3265. 
128. Liu L, Li P, Liu Y, Zhang Y. Efficacy of Probiotics and Synbiotics in Patients with Nonalcoholic Fatty Liver Disease: A MetaAnalysis. Dig Dis Sci. 2019;64(12):3402-3412.

129. Roychowdhury S, Glueck B, Han Y, Mohammad MA, Cresci GAM. A Designer Synbiotic Attenuates Chronic-Binge EthanolInduced Gut-Liver Injury in Mice. Nutrients. 2019;11(1):97.

130. Han Y, Glueck B, Shapiro D, Miller A, Roychowdhury S, Cresci GAM. Dietary Synbiotic Supplementation Protects Barrier Integrity of Hepatocytes and Liver Sinusoidal Endothelium in a Mouse Model of Chronic-Binge Ethanol Exposure. Nutrients. 2020;12(2):373.

131. Sittiprapaporn P, Sirilun S, Chaiyasut C, et al. The effect of synbiotics supplement on alcohol use disorders identification test and biochemical parameters, gamma glutamyl transferase, lipopolysaccharide and immunoglobulin a levels, in high risk alcoholics. Asian Journal of Medical Sciences. 2020;11(1):1-6.

132. Liu Q, Duan ZP, Ha DK, Bengmark S, Kurtovic J, Riordan SM. Synbiotic modulation of gut flora: effect on minimal hepatic encephalopathy in patients with cirrhosis. Hepatology. 2004;39(5):1441-1449.

133. Kühn F, Duan R, Ilmer M, et al. Targeting the Intestinal Barrier to Prevent Gut-Derived Inflammation and Disease: A Role for Intestinal Alkaline Phosphatase. Visceral Medicine. 2021;5(6):1-11.

134. Eliakim R, Mahmood A, Alpers DH. Rat intestinal alkaline phosphatase secretion into lumen and serum is coordinately regulated. Biochim Biophys Acta. 1991;1091(1):1-8.

135. Moss AK, Hamarneh SR, Mohamed MM, et al. Intestinal alkaline phosphatase inhibits the proinflammatory nucleotide uridine diphosphate. Am J Physiol Gastrointest Liver Physiol. 2013;304(6):G597-604.

136. Molnar K, Vannay A, Szebeni B, et al. Intestinal alkaline phosphatase in the colonic mucosa of children with inflammatory bowel disease. World J Gastroenterol. 2012;18(25):3254-3259.

137. Tuin A, Poelstra K, de Jager-Krikken A, et al. Role of alkaline phosphatase in colitis in man and rats. Gut. 2009;58(3):379-387.

138. Ramasamy S, Nguyen DD, Eston MA, et al. Intestinal alkaline phosphatase has beneficial effects in mouse models of chronic colitis. Inflamm Bowel Dis. 2011;17(2):532-542.
139. Heemskerk S, Masereeuw R, Moesker O, et al. Alkaline phosphatase treatment improves renal function in severe sepsis or septic shock patients. Crit Care Med. 2009;37(2):417-423, e411.

140. Kats S, Brands R, Seinen W, et al. Anti-inflammatory effects of alkaline phosphatase in coronary artery bypass surgery with cardiopulmonary bypass. Recent Pat Inflamm Allergy Drug Discov. 2009;3(3):214-220.

141. Mizumori M, Ham M, Guth PH, Engel E, Kaunitz JD, Akiba Y. Intestinal alkaline phosphatase regulates protective surface microclimate $\mathrm{pH}$ in rat duodenum. $J$ Physiol. 2009;587(Pt 14):3651-3663.

142. Liu W, Hu D, Huo H, et al. Intestinal Alkaline Phosphatase Regulates Tight Junction Protein Levels. J Am Coll Surg. 2016;222(6):1009-1017.

143. Hamarneh SR, Mohamed MMR, Economopoulos KP, et al. A Novel Approach to Maintain Gut Mucosal Integrity Using an Oral Enzyme Supplement. Annals of Surgery. 2014;260(4):706-715.

144. Malo MS, Alam SN, Mostafa G, et al. Intestinal alkaline phosphatase preserves the normal homeostasis of gut microbiota. Gut. 2010;59(11):1476-1484.

145. Kühn F, Adiliaghdam F, Cavallaro PM, et al. Intestinal alkaline phosphatase targets the gut barrier to prevent aging. JCI Insight. 2020;5(6):e134049.

146. Liu Y, Cavallaro PM, Kim B-M, et al. A role for intestinal alkaline phosphatase in preventing liver fibrosis. Theranostics. 2021;11(1):14-26.

147. Hamarneh SR, Kim BM, Kaliannan K, et al. Intestinal Alkaline Phosphatase Attenuates Alcohol-Induced Hepatosteatosis in Mice. Dig Dis Sci. 2017;62(8):2021-2034.

Publisher's Note Springer Nature remains neutral with regard to jurisdictional claims in published maps and institutional affiliations. 